\title{
Applications of certain linear operators in the theory of analytic functions
}

\author{
by H. M. SRivastava (Victoria, B.C.)
}

\begin{abstract}
The object of the present paper is to illustrate the usefulness, in the theory of analytic functions, of various linear operators which are defined in terms of (for example) fractional derivatives and fractional integrals, Hadamard product or convolution, and so on.
\end{abstract}

1. Introduction and definitions. Let $\mathcal{A}$ denote the class of functions normalized by

$$
f(z)=z+\sum_{n=2}^{\infty} a_{n} z^{n}
$$

which are analytic in the open unit disk

$$
\mathcal{U}=\{z:|z|<1\}
$$

Also let $\mathcal{S}$ denote the class of all functions in $\mathcal{A}$ which are univalent in $\mathcal{U}$. We denote by $\mathcal{S}^{*}(\alpha)$ and $\mathcal{K}(\alpha)$ the subclasses of $\mathcal{S}$ consisting of all functions which are, respectively, starlike and convex of order $\alpha$ in $\mathcal{U}(0 \leq \alpha<1)$, that is,

$$
\begin{aligned}
& (1.2) \quad \mathcal{S}^{*}(\alpha)=\left\{f: f \in \mathcal{S} \text { and } \operatorname{Re}\left(\frac{z f^{\prime}(z)}{f(z)}\right)>\alpha(0 \leq \alpha<1 ; z \in \mathcal{U})\right\}, \\
& (1.3) \quad \mathcal{K}(\alpha)=\left\{f: f \in \mathcal{S} \text { and } \operatorname{Re}\left(1+\frac{z f^{\prime \prime}(z)}{f^{\prime}(z)}\right)>\alpha(0 \leq \alpha<1 ; z \in \mathcal{U})\right\} .
\end{aligned}
$$

It follows readily from (1.2) and (1.3) that

$$
f(z) \in \mathcal{K}(\alpha) \Leftrightarrow z f^{\prime}(z) \in \mathcal{S}^{*}(\alpha) \quad(0 \leq \alpha<1),
$$

whose special case, when $\alpha=0$, is the familiar Alexander theorem (cf., e.g., $[2$, p. 43, Theorem 2.12]). We note also that

$$
\mathcal{K}(\alpha) \subset \mathcal{S}^{*}(\alpha) \subset \mathcal{S} \quad(0 \leq \alpha<1),
$$

1980 Mathematics Subject Classification (1985 Revision): Primary 30C45; Secondary 26А33, 33А 30. 


$$
\begin{array}{ll}
\mathcal{S}^{*}(\alpha) \subseteq \mathcal{S}^{*}(0) \equiv \mathcal{S}^{*} & (0 \leq \alpha<1), \\
\mathcal{K}(\alpha) \subseteq \mathcal{K}(0) \equiv \mathcal{K} & (0 \leq \alpha<1) .
\end{array}
$$

For the functions $f_{j}(z)$ defined by

$$
f_{j}(z)=\sum_{n=0}^{\infty} a_{j, n+1} z^{n+1} \quad(j=1,2),
$$

we denote by $f_{1} * f_{2}(z)$ the Hadamard product or convolution of the functions $f_{1}(z)$ and $f_{2}(z)$, that is,

$$
f_{1} * f_{2}(z)=\sum_{n=0}^{\infty} a_{1, n+1} a_{2, n+1} z^{n+1} .
$$

Thus, following Ruscheweyh [9], a function $f(z) \in \mathcal{A}$ is said to be prestarlike of order $\alpha(\alpha \leq 1)$ if and only if

$$
\begin{cases}\frac{z}{(1-z)^{2(1-\alpha)}} * f(z) \in \mathcal{S}^{*}(\alpha) & (\alpha<1), \\ \operatorname{Re}\left(\frac{f(z)}{z}\right)>\frac{1}{2} & (\alpha=1),\end{cases}
$$

and we denote by $\mathcal{R}(\alpha)$ the subclass of $\mathcal{A}$ consisting of all prestarlike functions of order $\alpha$ in $\mathcal{U}$.

The various linear operators (whose usefulness, in the theory of such subclasses of analytic functions as those defined above, will be considered in this paper) include the Carlson-Shaffer operator $\mathcal{L}(a, c)$ defined by (cf. [1])

$$
\mathcal{L}(a, c) f(z)=\varphi(a, c ; z) * f(z) \quad(f(z) \in \mathcal{U}),
$$

where $\varphi(a, c ; z)$ is an incomplete Beta function defined by

$$
\begin{aligned}
\varphi(a, c ; z)=\sum_{n=0}^{\infty} \frac{(a)_{n}}{(c)_{n}} z^{n+1}=z{ }_{2} F_{1}(1, a ; c ; z) \\
\quad(c \neq 0,-1,-2, \ldots ; z \in \mathcal{U}),
\end{aligned}
$$

in terms of the Pochhammer symbol $(\lambda)_{n}$ given by

$$
(\lambda)_{n}=\frac{\Gamma(\lambda+n)}{\Gamma(\lambda)}= \begin{cases}1 & (n=0) \\ \lambda(\lambda+1) \ldots(\lambda+n-1) & (n \in \mathbb{N}=\{1,2,3, \ldots\}),\end{cases}
$$

and of the Gaussian case $p-1=q=1$ of the generalized hypergeometric function ${ }_{p} F_{q}(z)$ defined, for complex parameters $\alpha_{j}(j=1, \ldots, p)$ and $\beta_{j}$ $(\neq 0,-1,-2, \ldots)(j=1, \ldots, q)$, by

$$
\begin{aligned}
{ }_{p} F_{q}(z) & \equiv{ }_{p} F_{q}\left(\alpha_{1}, \ldots, \alpha_{p} ; \beta_{1}, \ldots, \beta_{q} ; z\right) \\
& ={ }_{p} F_{q}\left[\begin{array}{c}
\alpha_{1}, \ldots, \alpha_{p} ; \\
\beta_{1}, \ldots, \beta_{q} ;
\end{array}\right]
\end{aligned}
$$




$$
\begin{aligned}
=\sum_{n=0}^{\infty} & \frac{\left(\alpha_{1}\right)_{n} \ldots\left(\alpha_{p}\right)_{n}}{\left(\beta_{1}\right)_{n} \ldots\left(\beta_{q}\right)_{n}} \frac{z^{n}}{n !} \quad(p \leq q+1) \\
& (p \leq q \text { and }|z|<\infty ; p=q+1 \text { and } z \in \mathcal{U}) .
\end{aligned}
$$

We note in passing that

$$
z_{p} F_{q}\left(\alpha_{1}, \ldots, \alpha_{p} ; \beta_{1}, \ldots, \beta_{q} ; z\right) \in \mathcal{A} .
$$

The Carlson-Shaffer operator $\mathcal{L}(a, c)$ maps $\mathcal{A}$ onto itself. Moreover, if $a \neq 0,-1,-2, \ldots$, then $\mathcal{L}(c, a)$ is an inverse of $\mathcal{L}(a, c)$. Observe also that (cf. [8, p. 1067])

$$
\mathcal{K}(\alpha)=\mathcal{L}(1,2) \mathcal{S}^{*}(\alpha) ; \quad \mathcal{S}^{*}(\alpha)=\mathcal{L}(2,1) \mathcal{K}(\alpha) \quad(0 \leq \alpha<1) .
$$

Next we recall the generalized Bernardi-Libera-Livingston integral operator $\mathcal{J}_{\gamma}$ defined by

$$
\mathcal{J}_{\gamma} f(z)=\frac{\gamma+1}{z^{\gamma}} \int_{0}^{z} t^{\gamma-1} f(t) d t \quad(\gamma>-1 ; f(z) \in \mathcal{A})
$$

which, for various further constraints on the parameter $\gamma$, was used recently by several authors (see, e.g., [15, pp. 66, 154, 181, and 338]).

Numerous operators of fractional calculus (that is, fractional integral and fractional derivative) have indeed been studied in the literature rather extensively (cf., e.g., [3, Chapter 13], [4], [5], [10], [11], [13, p. 28 et seq.], [14, Chapter 5], and [15]). We choose to recall here the following interesting generalization of the fractional derivative operator $D_{z}^{\lambda}$ (considered by Owa [6]):

Definition (cf. [7] and [12]). For real numbers $\lambda, \mu$, and $\nu$, the fractional derivative operator $J_{0, z}^{\lambda, \mu, \nu}$ is defined by

$$
\begin{aligned}
J_{0, z}^{\lambda, \mu, \nu} f(z)=\frac{1}{\Gamma(1-\lambda)} & \frac{d}{d z}\left\{z^{\lambda-\mu} \int_{0}^{z}(z-\zeta)^{-\lambda}\right. \\
& \left.\times{ }_{2} F_{1}(\mu-\lambda,-\nu ; 1-\lambda ; 1-\zeta / z) f(\zeta) d \zeta\right\} \\
& (0 \leq \lambda<1 ; \kappa>\max \{0, \mu-\nu-1\}-1),
\end{aligned}
$$

where $f(z)$ is an analytic function in a simply-connected region of the $z$ plane containing the origin, with the order

$$
f(z)=O\left(|z|^{\kappa}\right) \quad(z \rightarrow 0)
$$

and the multiplicity of $(z-\zeta)^{-\lambda}$ is removed by requiring $\log (z-\zeta)$ to be real when $z-\zeta>0$.

It follows readily from the definition (1.17) that

$$
J_{0, z}^{\lambda, \lambda, \nu} f(z)=D_{z}^{\lambda} f(z) \quad(0 \leq \lambda<1),
$$


where the fractional calculus operator $D_{z}^{\lambda}$ is, in fact, defined for all values of $\lambda$ (see, e.g., [15, p. 343]).

2. Applications involving univalent and starlike generalized hypergeometric functions. We begin by recalling the following inclusion theorem for the generalized hypergeometric function, which was proven by Owa and Srivastava [8] by applying the Carlson-Shaffer operator $\mathcal{L}(a, c)$ :

THEOREM 1. For the generalized hypergeometric function ${ }_{p} F_{q}(z)$ defined by (1.13), let

$$
\begin{aligned}
&\left|\frac{z{ }_{p} F_{q}^{\prime \prime}(z)}{{ }_{p} F_{q}^{\prime}(z)}\right|<(1-\alpha)^{-1}\left(1-\frac{3}{2} \alpha+\alpha^{2}\right) \\
&\left(0 \leq \alpha \leq 1 / 2 ; \alpha_{1} \ldots \alpha_{p} \neq 0 ; \quad z \in \mathcal{U}\right) .
\end{aligned}
$$

Then

$$
\begin{array}{r}
z_{p+1} F_{q+1}\left(\alpha_{1}+1, \ldots, \alpha_{p}+1,1 ; \beta_{1}+1, \ldots, \beta_{q}+1,2 ; z\right) \in \mathcal{S}^{*}(\alpha) \\
\left(0 \leq \alpha \leq \frac{1}{2}\right)
\end{array}
$$

Now, in view of the fact that

$$
f(z) \in \mathcal{S}^{*} \Rightarrow \mathcal{J}_{\gamma} f(z) \in \mathcal{S}^{*} \quad(0 \leq \gamma \leq 1),
$$

Theorem 1 (with $\alpha=0$ ) and the definition (1.16) would lead us eventually to

THEOREM 2. For the generalized hypergeometric function ${ }_{p} F_{q}(z)$ defined by (1.13), let

$$
\left|\frac{z_{p} F_{q}^{\prime \prime}(z)}{{ }_{p} F_{q}^{\prime}(z)}\right|<1 \quad\left(\alpha_{1} \ldots \alpha_{p} \neq 0 ; z \in \mathcal{U}\right) .
$$

Then

$$
\begin{array}{r}
z_{p+s+1} F_{q+s+1}\left[\begin{array}{r}
\alpha_{1}+1, \ldots, \alpha_{p}+1,1, \gamma_{1}+1, \ldots, \gamma_{s}+1 ; \\
\beta_{1}+1, \ldots, \beta_{q}+1,2, \gamma_{1}+2, \ldots, \gamma_{s}+2 ;
\end{array}\right] \in \mathcal{S}^{*} \\
\left(0 \leq \gamma_{j} \leq 1 ; j=1, \ldots, s\right) .
\end{array}
$$

From among various special cases of Theorem 2, which are worthy of note, we mention here the case when $\gamma_{j}=1(j=1, \ldots, s)$. The assertion (2.5) reduces, in this special case, to the inclusion relation:

$$
z_{p+s} F_{q+s}\left[\begin{array}{c}
\alpha_{1}+1, \ldots, \alpha_{p}+1,1,2, \ldots, 2 ; \\
\beta_{1}+1, \ldots, \beta_{q}+1,3,3, \ldots, 3 ;
\end{array}\right] \in \mathcal{S}^{*},
$$

which holds true under the relevant hypotheses of Theorem 2 . 
3. An application involving the classes $\mathcal{R}(\alpha)$ and $\mathcal{K}(\alpha)$. It is easily seen from the definition (1.9) for the class $\mathcal{R}(\alpha)$ that

$$
\mathcal{R}(\alpha)=\mathcal{L}(1,2-2 \alpha) \mathcal{S}^{*}(\alpha) \quad(\alpha<1)
$$

and

$$
\mathcal{R}(1)=\left\{f: f \in \mathcal{A} \text { and } \operatorname{Re}(f(z) / z)>\frac{1}{2}(z \in \mathcal{U})\right\} .
$$

With a view to presenting a connection theorem involving the classes $\mathcal{R}(\alpha)$ and $\mathcal{K}(\alpha)$, we find it to be convenient to introduce the operator $\Omega_{z}^{\lambda, \mu, \nu}$ defined by

$$
\Omega_{z}^{\lambda, \mu, \nu} f(z)=\frac{\Gamma(2-\mu) \Gamma(3-\lambda+\nu)}{\Gamma(3-\mu+\nu)} z^{\mu} J_{0, z}^{\lambda, \mu, \nu} f(z) \quad(f(z) \in \mathcal{A}),
$$

where $J_{0, z}^{\lambda, \mu, \nu}$ denotes the fractional derivative operator defined already by (1.17). Since

$$
J_{0, z}^{\lambda, \mu, \nu} z^{\rho}=\frac{\Gamma(\rho+1)(\rho-\mu+\nu+2)}{\Gamma(\rho-\mu+1) \Gamma(\rho-\lambda+\nu+2)} z^{\rho-\mu} \quad(\rho+2>\mu-\nu),
$$

which stems naturally from the definition (1.17), it is not difficult to relate the operators $\Omega_{z}^{\lambda, \mu, \nu}$ and $\mathcal{L}(a, c)$ as follows:

$$
\begin{array}{r}
\Omega_{z}^{\lambda, \mu, \nu} f(z)=\mathcal{L}(2,2-\mu) \mathcal{L}(3-\mu+\nu, 3-\lambda+\nu) f(z) \\
(0 \leq \lambda<1 ; \mu-\nu<3 ; f(z) \in \mathcal{A}) .
\end{array}
$$

Making use of the relationships (3.5) and (1.15), and the following result (due essentially to Carlson and Shaffer [1]):

$$
\mathcal{L}(2-2 \beta, 2-2 \alpha) \mathcal{S}^{*}(\alpha) \subset \mathcal{S}^{*}(\beta) \subset \mathcal{S}^{*}(\alpha) \quad(0 \leq \alpha \leq \beta<1),
$$

it can be shown that

$$
\begin{aligned}
\mathcal{L}(3-\lambda+\nu, 3-\mu+\nu) \Omega_{z}^{\lambda, \mu, \nu} & \mathcal{K}(1 / 2) \subset \mathcal{S}^{*}(1 / 2) \\
& (0 \leq \lambda<1 ; \mu-\nu<3 ; 0 \leq \mu<1) .
\end{aligned}
$$

Finally, rewriting a special case of $(3.6)$ in the form:

$$
\mathcal{L}(1,2-\alpha) \mathcal{S}^{*}(\alpha / 2) \subset \mathcal{S}^{*}(1 / 2) \subset \mathcal{S}^{*}(\alpha / 2) \quad(0 \leq \alpha<2),
$$

we obtain the following connection theorem involving the classes $\mathcal{R}(\alpha)$ and $\mathcal{K}(\alpha)$ :

TheOREM 3. For the classes $\mathcal{K}(\alpha)$ and $\mathcal{R}(\alpha)$ defined by (1.3) and (1.9), respectively,

$$
\begin{aligned}
\mathcal{L}(3-\lambda+\nu, 3-\mu+\nu) \Omega_{z}^{\lambda, \mu, \nu} & \mathcal{K}(\mu / 2)=\mathcal{R}(\mu / 2) \\
& (0 \leq \lambda<1 ; \mu-\nu<3 ; 0 \leq \mu<2) .
\end{aligned}
$$

For $\mu=0$ and $\mu=1$, the assertion (3.9) of Theorem 3 simplifies considerably. The details may be omitted. 
Acknowledgements. The author wishes to thank the Polish Academy of Sciences and Łódź University for having invited and supported his visits to their Institutes of Mathematics and Physics, as well as his participation in the Tenth International Conference on Analytic Functions held at Szczyrk on April 22-27, 1990. This investigation was supported, in part, by the Natural Sciences and Engineering Research Council of Canada under Grant OGP0007353.

\section{References}

[1] B. C. Carlson and D. B. Shaffer, Starlike and prestarlike hypergeometric functions, SIAM J. Math. Anal. 15 (1984), 737-745.

[2] P. L. Duren, Univalent Functions, Grundlehren Math. Wiss. 259, Springer, New York 1983.

[3] A. Erdélyi, W. Magnus, F. Oberhettinger, and F. G. Tricomi, Tables of Integral Transforms, Vol. II, McGraw-Hill, New York 1954.

[4] K. Nishimoto, Fractional Calculus, Vols. I, II, and III, Descartes Press, Koriyama 1984, 1987, and 1989.

[5] K. B. Oldham and J. Spanier, The Fractional Calculus: Theory and Applications of Differentiation and Integration to Arbitrary Order, Academic Press, New YorkLondon 1974.

[6] S. Owa, On the distortion theorems. II, Kyungpook Math. J. 18 (1978), 53-59.

[7] S. Owa, M. Saigo, and H. M. Srivastava, Some characterization theorems for starlike and convex functions involving a certain fractional integral operator, J. Math. Anal. Appl. 140 (1989), 419-426.

[8] S. Owa and H. M. Srivastava, Univalent and starlike generalized hypergeometric functions, Canad. J. Math. 39 (1987), 1057-1077.

[9] S. Ruscheweyh, Linear operators between classes of prestarlike functions, Comment. Math. Helv. 52 (1977), 497-509.

[10] M. Saigo, A remark on integral operators involving the Gauss hypergeometric functions, Math. Rep. College General Ed. Kyushu Univ. 11 (1978), 135-145.

[11] S. G. Samko, A. A. Kilbas, and O. I. Marichev, Integrals and Derivatives of Fractional Order and Some of Their Applications, Nauka i Tekhnika, Minsk 1987 (in Russian).

[12] N. S. Sohi, Distortion theorems involving certain operators of fractional calculus on a class of p-valent functions, in: Fractional Calculus and Its Applications, K. Nishimoto (ed.), College of Engineering (Nihon University), Koriyama 1990, 245252.

[13] H. M. Srivastava and R. G. Buschman, Convolution Integral Equations with Special Function Kernels, Halsted Press, Wiley, New York 1977.

[14] H. M. Srivastava and H. L. Manocha, A Treatise on Generating Functions, Halsted Press, Wiley, New York 1984.

[15] H. M. Srivastava and S. Owa (eds.), Univalent Functions, Fractional Calculus, and Their Applications, Halsted Press, Wiley, New York 1989.

DEPARTMENT OF MATHEMATICS AND STATISTICS

UNIVERSITY OF VICTORIA

VICTORIA, BRITISH COLUMBIA V8W 3P4

CANADA

Reçu par la Rédaction le 14.9.1990 